\title{
Are intraoperative precursor events associated with postoperative major adverse events?
}

\author{
Christine R. Herman, MD, ${ }^{\mathrm{a}, \mathrm{b}}$ Jean-François Légaré, MD, ${ }^{\mathrm{a}}$ Adrian Levy, PhD, ${ }^{\mathrm{b}}$ Karen J. Buth, MSc, ${ }^{\mathrm{a}}$ and \\ Roger Baskett, $\mathrm{MD}^{\mathrm{a}}$
}

Objectives: Precursor events are undesirable events that can lead to a subsequent adverse event and have been associated with postoperative mortality. The purpose of the present study was to determine whether precursor events are associated with a composite endpoint of major adverse cardiac events (MACE) (death, acute renal failure, stroke, infection) in a low- to medium-risk coronary artery bypass grafting, valve, and valve plus coronary artery bypass grafting population. These events might be targets for strategies aimed at quality improvement.

\begin{abstract}
Methods: The present study was a retrospective cohort design performed at the Queen Elizabeth Health Science Centre. Low- to medium-risk patients who had experienced postoperative MACE were matched 1:1 with patients who had not experienced postoperative MACE. The operative notes, for both groups, were scored by 5 surgeons to determine the frequency of 4 precursor events: bleeding, difficulty weaning from cardiopulmonary bypass, repair or regrafting, and incomplete revascularization or repair. A univariate comparison of $\geq 1$ precursor events in the matched groups was performed.
\end{abstract}

Results: A total of 311 MACE patients $(98.4 \%)$ were matched. The primary outcome occurred more frequently in the MACE group than in the non-MACE group $(33 \%$ vs $24 \% ; P=.015)$. The incidence of the individual events of bleeding and difficulty weaning from cardiopulmonary bypass was significantly higher in the MACE group. Those patients with a precursor event in the absence of MACE also appeared to have a greater prevalence of other important postoperative outcomes.

Conclusions: Patients undergoing cardiac surgery who are exposed to intraoperative precursor events were more likely to experience a postoperative MACE. Quality improvement techniques aimed at mitigating the consequences of precursor events might improve the surgical outcomes for cardiac surgical patients. (J Thorac Cardiovasc Surg 2014;147:1499-504)

An important component of quality improvement in cardiac surgery is an impartial evaluation of death and major morbidity in patients undergoing cardiac surgery. These metrics serve as quality indicators for outcomes, process, and structure of care. ${ }^{1,2}$ Specifically, preventable death and morbidity are logical targets for quality improvement strategies, because they are potentially avoidable. Unavoidable injury, however, is thought to occur unexpectedly, with the temporal sequence of the inciting event to the injury very short. The published data have suggested that $56 \%$ to $87 \%$ of deaths in cardiac patients are nonpreventable, ${ }^{3,4}$ potentially altering the success of quality improvement efforts.

\footnotetext{
From the Division of Cardiac Surgery ${ }^{\mathrm{a}}$ and Department of Community Health and Epidemiology, ${ }^{\mathrm{b}}$ Dallhousie University, Halifax, Nova Scotia, Canada.

Disclosures: Authors have nothing to disclose with regard to commercial support.

Received for publication Oct 12, 2012; revisions received Jan 21, 2013; accepted for publication May 30, 2013; available ahead of print July 18, 2013.

Address for reprints: Christine R. Herman, MD, Division of Cardiac Surgery, Dallhousie University, 1796 Summer St, Halifax, Nova Scotia B3H 3A7, Canada (E-mail: cherman@dal.ca). 0022-5223/\$36.00

Crown Copyright $\odot 2014$ Published by Elsevier Inc. on behalf of The American Association for Thoracic Surgery

http://dx.doi.org/10.1016/j.jtcvs.2013.05.018
}

It is possible that the causal pathway of these "unavoidable" injuries begins long before any overt warning signs are present. Upstream events, such as precursor events, have received recent attention as a focus for alterability. Precursor events are events that precede an adverse event such as death. ${ }^{5}$ They are undesirable events that can lead to a subsequent adverse event. Intraoperative precursor events have been reported to occur in $73.3 \%$ of cardiac cases, with a mean of 3.5 precursor events per case. ${ }^{6}$ Also, precursor events occurred more frequently in patients experiencing "death or near miss." Intraoperative near misses and major complications have also been associated with increased postoperative death, ${ }^{7,8}$ especially if the event went uncompensated.

Because compensation or appropriate responses to critical intraoperative events are important to prevent a potential adverse event from subsequently developing, ${ }^{9}$ a surgical team might believe that a precursor event, if compensated for, would negate this potential. However, it might be, rather, that these "forgotten" events, whether compensated for or not, are the nidus in which begins the propagation of the precursor event toward an adverse event. Usually, such propagation is prevented through system safeguards ${ }^{10}$; 


\section{Abbreviations and Acronyms \\ $\mathrm{CABG}=$ coronary artery bypass grafting \\ $\mathrm{CPB}=$ cardiopulmonary bypass \\ MACE $=$ major adverse cardiac events \\ $\mathrm{OR} \quad=$ operating room}

however, failure to recognize the importance of the precursor event could act as a "hole" in this layer of defense. As such, even nominal events could pose a potential risk to the patient and could account for death or major injury, regardless of the perceived "preventability."

The goal of the present study was to determine whether intraoperative precursor events, regardless of the compensation or seriousness, contribute to postoperative death or major morbidity. In the present study, we chose to emphasize the coronary artery bypass grafting (CABG), valve, and CABG plus valve patient population, because this patient group encompasses most of current cardiac surgery clinical practice. Also, we limited the risk profile to low to medium preoperative predicted risk, because high-risk patients represent a small proportion of clinical practice, and their acuity might overwhelm any effect precursor events would have on subsequent outcomes.

\section{METHODS \\ Data Source}

The present study was a retrospective cohort design. The Maritime Heart Center Cardiac Surgery Registry is a detailed clinical database housed at the Queen Elizabeth II Health Science Center (Halifax, Nova Scotia, Canada). It includes pre-, intra-, and postoperative data prospectively collected for all cardiac surgical cases performed at the Queen Elizabeth II Health Science Center from 1995 to the present. The data are collected by trained abstractors, and a database administrator maintains the registry. The database is audited annually.

\section{Study Population}

A patient cohort from 2004 to 2009 that included consecutive CABG, aortic valve replacement, mitral valve repair, and mitral valve replacement with or without CABG was used to develop a nonparsimonious logistic regression model that described the composite outcome of major adverse cardiac events (MACE). MACE was defined as in-hospital death, stroke (permanent or transient), acute renal failure ( $>176 \mathrm{mmol}$ or a $50 \%$ increase from baseline if chronic renal failure), or infection (sepsis, pneumonia, or deep sternal wound infection). The predictor variables included in the model were important preoperative variables that might influence the probability of experiencing postoperative MACE. A detailed description of the model development can found in the Appendix.

The predicted probability of MACE was calculated for each patient in the model cohort. To define a study cohort of patients with low to medium risk of MACE, the model cohort was truncated at the 75th percentile. Using the predicted probability of MACE, each patient with postoperative MACE was matched 1:1 with a patient without postoperative MACE. A greedy matched algorithm that proceeded from a 5-digit to a 1-digit match on risk was used.

\section{Precursor Events}

Precursor events were defined as undesirable incidents that occurred proximally in the causal pathway of an adverse event. They are required for the adverse event to occur but do not always result in said event. As such, precursor events will occur with more frequency than will adverse events. ${ }^{5}$ In general, precursor events are easily compensated for by the care team and are seldom recognized as a critical event in the causal pathway of an adverse event. Four intraoperative precursor events of interest were identified through quality assessment exercises at our institution: (1) bleeding, (2) failure to wean from cardiopulmonary bypass (CPB), (3) regrafting or repair of conduit or valve, and (4) incomplete revascularization or repair.

Bleeding was defined as any instance of surgical bleeding (other than related to the grafts) or coagulopathy requiring intervention such as repair, a return to CPB for repair, administration of blood products, or a delay in the termination of surgery. Failure to wean from CPB included any instance in which the patient did not wean or struggled to wean, requiring a return to $\mathrm{CPB}$, insertion of an intra-aortic balloon pump, additional inotropic support, insertion of a ventricular assist device, and pacing or prewean pacing required to wean in the specific instance of valve surgery. Regrafting or repair of the conduit or valve included any instance in which regrafting or repair was undertaken because of issues with conduit length, lie, poor flow reading, wall motion abnormality on transesophageal echocardiogram, bleeding, perivalvular leakage, and persistent stenosis or regurgitation despite repair or replacement. Incomplete revascularization or repair occurred when not all critically diseased territories had undergone grafting or persistent stenosis or regurgitation or perivalvular leakage considered significant by best standard of care practices was not corrected. This could have been because of an inadequate size of the target vessel, a paucity of conduit, or other patient or technical factors.

The primary outcome was having $\geq 1$ intraoperative precursor events.

The operative notes were used to identify the precursor events according to the surgeons' description of the events in the operating room (OR). These operative notes were dictated immediately after the operation before a MACE occurred, allowing for an unbiased measure of the prevalence of the precursor events. Patient and surgeon identifiers were removed from the operative notes for the matched groups, and the anonymous operative notes were distributed to 5 surgeons for review and identification of the precursor events. No surgeon received their own operative notes. Any operative note that reported a death in the OR or that was reported as a late dictation was removed from the review, along with the matched partner note. Inter-rater reliability was assessed.

A secondary analysis was planned to examine whether the precursor events were associated with other secondary outcomes, including pump time, clamp time, intraoperative inotropic use, postoperative blood product use, low cardiac output syndrome, and ventilator and intensive care unit length of stay. This was achieved by excluding the MACE events from the matched groups and then comparing all patients with a precursor event with those who did not. This was a hypothesis-generating analysis only.

\section{Statistical Analysis}

Previous research from our institution examining precursor events and death in a low-risk CABG population revealed that patients who died postoperatively had had 30\% more precursor events than those who did not die. For the study to achieve $80 \%$ power with an alpha of 0.05 , the required sample size per group for a $50 \%, 40 \%$, and $30 \%$ relative risk difference was 48,112 , and 243 , respectively. Because the present study had a mixed population with an unknown risk difference, the largest sample size was used.

Continuous variables were compared using a 2-tailed $t$ test or Wilcoxon rank sum test, and categorical variables were analyzed using a chi-square or Fisher's exact test, as appropriate. The prevalence of precursor events was compared between the matched groups (those with MACE vs those without MACE). The outcomes were analyzed using the chi-square test or Fisher's exact test, as appropriate.

All statistical analysis was performed using Statistical Analysis Systems software, version 9.2 (SAS Institute, Cary, NC). 
The institutional review board of the Capital District Health Authority approved the present study. The requirement to obtain informed consent was waived under Section 2.1c of the Tri-Council Policy Statement. All personal identifiers were stripped before data analysis to ensure patient anonymity and confidentiality.

\section{RESULTS}

\section{Study Population}

The model derivation cohort included 4270 consecutive patients and 17 variables (Table 2 ). The predicted probability of MACE was calculated using the model and assigned to each patient. To create a low- to medium-risk group, the cohort was truncated at the 75 th percentile of predicted risk $(n=3192)$. The mean predicted probability for the entire cohort and the low- to medium-risk cohort was 10.5 (interquartile range [IQR], 6.1-20.2) and 8 (IQR, 5.4-12), respectively. The low- to medium-risk group experienced 316 MACE events (9.9\%).

A total of 311 patients $(98.4 \%)$ with MACE events were matched 1:1 (Figure 1). Of these 311 patients, $25(8 \%)$ and their match were eliminated from the analysis because the patient had died in the OR $(\mathrm{n}=2)$, the dictation was reported as late $(n=12)$, or the OR dictation was not found $(\mathrm{n}=9)$. The preoperative clinical characteristics were similar between the matched MACE $(\mathrm{n}=286)$ and non-MACE $(\mathrm{n}=286)$ cases available for analysis (Table 1$)$. The predicted probability of MACE was almost identical in the non-MACE (11.9; IQR, 0.084-17.90) and MACE (11.9; IQR, 0.08-17.98) groups.

Five surgeons reviewed and identified the precursor events in all cases.

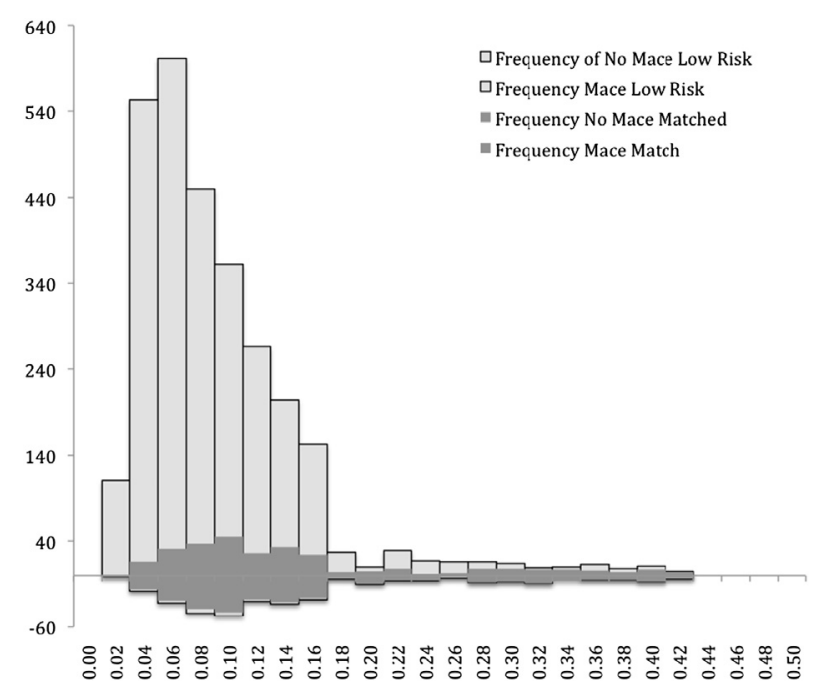

FIGURE 1. Histogram of matched major adverse cardiac events (MACE) cases compared with the total population. The light columns on the upper and lower axes indicate the total number of cases without (upper axis) and with (lower axis) MACE. The dark columns indicate the proportion of matched non-MACE cases (upper axis) to MACE cases (lower axis). The light blues columns represent all the matched cases.
TABLE 1. Preoperative variables in the matched groups (no MACE vs MACE)

\begin{tabular}{|c|c|c|c|}
\hline Variable & No MACE $(n=286)$ & $\operatorname{MACE}(n=286)$ & $P$ value \\
\hline Predicted probability & $11.9(0.084-17.90)^{*}$ & $11.9(0.08-17.98)^{*}$ & \\
\hline Age $(y)$ & $67(60-74) *$ & $68(60-75)^{*}$ & \\
\hline Female gender & 23.78 & 24.48 & .845 \\
\hline BMI $\left(\mathrm{kg} / \mathrm{m}^{2}\right)$ & & & .0982 \\
\hline$<25$ & 16.78 & 24.13 & \\
\hline $25-30$ & 39.51 & 37.06 & \\
\hline $30-35$ & 30.07 & 23.78 & \\
\hline$>35$ & 13.64 & 15.03 & \\
\hline Diabetes & 38.11 & 38.81 & .8635 \\
\hline Hypertension & 74.13 & 77.62 & .3284 \\
\hline $\mathrm{AF}$ & 10.49 & 11.54 & .6887 \\
\hline COPD & 17.83 & 17.83 & 9999 \\
\hline CVD & 13.99 & 13.29 & .8075 \\
\hline PVD & 15.03 & 15.73 & .8167 \\
\hline Frailty & 2.8 & 2.8 & .9999 \\
\hline $\mathrm{EF}<40 \%$ & 13.99 & 12.24 & .5357 \\
\hline NYHA class & & & .6038 \\
\hline I & 26.92 & 30.07 & \\
\hline II & 24.13 & 25.52 & \\
\hline III & 35.66 & 30.42 & \\
\hline IV & 13.29 & 13.99 & \\
\hline Hemoglobin $(\mathrm{g} / \mathrm{L})$ & & & .9225 \\
\hline$<115$ & 51.05 & 51.75 & \\
\hline $115-135$ & 40.21 & 38.81 & \\
\hline$>135$ & 8.74 & 9.44 & \\
\hline Creatinine (mg/dL) & & & .9332 \\
\hline$<115$ & 75.17 & 76.22 & \\
\hline $115-140$ & 15.73 & 14.69 & \\
\hline $140-160$ & 3.85 & 4.55 & \\
\hline$>160$ & 5.24 & 4.55 & \\
\hline Redo surgery & 9.09 & 6.29 & .2094 \\
\hline Status & & & .5915 \\
\hline Elective & 49.3 & 51.05 & \\
\hline In-house & 43.36 & 41.96 & \\
\hline Urgent & 6.64 & 5.24 & \\
\hline Emergent & 0.7 & 1.75 & \\
\hline Procedure type & & & .9403 \\
\hline CABG & 57.34 & 55.94 & \\
\hline Valve & 13.64 & 14.34 & \\
\hline CABG plus valve & 29.02 & 29.72 & \\
\hline Surgeon & & & .6071 \\
\hline A & 10.49 & 14.34 & \\
\hline B & 11.19 & 11.54 & \\
\hline $\mathrm{C}$ & 9.79 & 11.89 & \\
\hline $\mathrm{D}$ & 2.45 & 2.45 & \\
\hline $\mathrm{E}$ & 9.79 & 8.04 & \\
\hline $\mathrm{F}$ & 9.79 & 9.79 & \\
\hline G & 3.15 & 3.85 & \\
\hline $\mathrm{H}$ & 19.93 & 16.08 & \\
\hline I & 9.09 & 11.89 & \\
\hline $\mathrm{J}$ & 14.34 & 10.14 & \\
\hline
\end{tabular}

MACE, Major adverse cardiac events; $B M I$, body mass index; $A F$, atrial fibrillation; $C O P D$, chronic obstructive pulmonary disease; $C V D$, cardiovascular disease; $P V D$, peripheral vascular disease; $E F$, ejection fraction; $N Y H A$, New York Heart Association; $C A B G$, coronary artery bypass grafting. *Interquartile range. 


\section{Primary Outcome}

The primary outcome of 1 or more precursor event occurred significantly more frequently in the MACE group $(33 \%$ vs $24 \% ; P=.015$; Figure 2$)$.

Each individual precursor event (ie, bleeding, difficulty weaning, repair or regrafting, and incomplete revascularization or repair) was identified in the MACE and non-MACE groups (Figure 2). The precursor events of bleeding and difficulty weaning contributed to the outcome the most, and repair or regrafting and incomplete revascularization occurred at similar frequencies in both the MACE and the non-MACE groups.

\section{Secondary Analysis}

When MACE was excluded, and the patients with precursor events were compared with the patients without precursor events, the pump and clamp time appeared similar and the intraoperative inotropic use, postoperative blood product use, and low cardiac output syndrome appeared to have greater prevalence. Patients with no precursor events had a similar prevalence of secondary outcomes as the total low- to medium-risk population (MACE excluded).

\section{DISCUSSION}

The objective of the present study was to describe the association of intraoperative precursor events and postoperative MACE. We found that cardiac surgery patients who are exposed to 1 or more intraoperative precursor event are more likely to experience postoperative MACE (33\% vs $24 \% ; P=.015)$. Individually, the bleeding and difficulty weaning from $\mathrm{CPB}$ precursor events appeared to contribute the most to the primary outcome.

Although hypothesis-generating only, the results of the secondary analysis suggested that other important outcomes (other than MACE) might be higher in patients exposed to

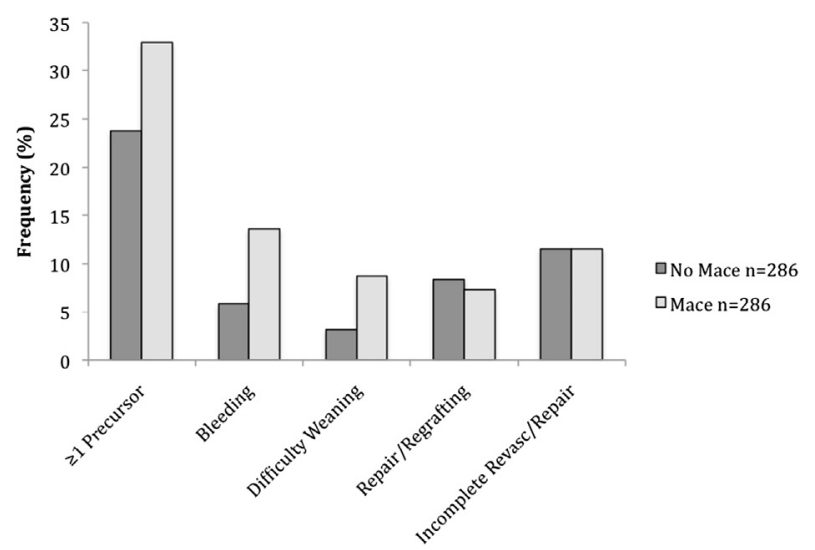

FIGURE 2. Frequency of precursor events for major adverse cardiac events (MACE) and non-MACE groups. The dark bars represent patients who did not experience a postoperative MACE and the light bars, those patients who did. a precursor event. Also, patients without precursor events had a similar prevalence of these events compared with the entire low- to medium-risk population (excluding MACE patients), indicating that they were representative of the entire low- to medium-risk population. Therefore, precursor events alone could be driving the greater prevalence of secondary outcomes in this group.

To understand how precursor events are associated with the development of an adverse event and how they might be altered, consideration of the structure of the adverse event is required. First, one should consider the layers of safeguards and system defenses that attempt to prevent adverse events. ${ }^{11,12}$ These layers of safeguards act as walls to block adverse events from occurring or propagating. The layers, however, are not always intact, with holes that can open or close and shift around. If the holes align, a direct path could develop, allowing the adverse event to occur. Next, one should consider the inciting event as a precursor event, or an error, preventable or not. These errors can be active or latent failures. ${ }^{11,13}$ Active failures are acts committed by people and could be a mistake or procedural violation that has immediately visible consequences. Latent failures have delayed consequences and are generally a result of an error-provoking environment or a gap in process. These latent failures can combine with other triggering events, each penetrating the layers of defense and "snowballing" to the final adverse event.

Active failures are generally compensated for immediately, and, as such, the surgical team might believe the event is no longer an issue. Approximately $90 \%$ of all precursor events have been reported as being adequately compensated $^{6}$; however, they were still associated with increased death or near miss. Also, the number of major precursor events per procedure and the number of precursor events per surgeon were independent predictors of death or near miss. This could indicate that compensation alone might be adequate to prevent downstream adverse events. Surgical teams might disregard precursor events once they have been deemed "compensated for," which starts the propagation through the system defenses, leading to the adverse event. Disregarding these events, in itself, might act as an important defect in the system defenses. Alternatively, if caused by a latent failure, the precursor event might overtly be inadequately compensated. Intraoperative near misses and major complications have also been associated with increased postoperative death, especially if the event was uncompensated in a cardiac population. ${ }^{7,8}$ As such, both forms of error or precursor events, regardless of compensation, can permeate the system defenses and lead to an adverse event.

This lack of a conscious association of precursor events with subsequent adverse events can further contribute to the propagation of the event, because increased attention to these patients is likely required to prevent or limit the propagation but might not be received by the patients. 
TABLE 2. Nonparsimonious logistic regression for MACE in a CABG/ valve population

\begin{tabular}{|c|c|c|}
\hline Variable & Odds ratio & $\mathbf{9 5} \% \mathrm{CI}$ \\
\hline Age square & 1.0 & $1.0-1.03$ \\
\hline Female gender & 0.8 & $0.6-1.0$ \\
\hline PVD & 1.6 & $1.2-1.9$ \\
\hline Frailty & 1.7 & $1.2-2.5$ \\
\hline \multicolumn{3}{|l|}{ BMI $\left(\mathrm{kg} / \mathrm{m}^{2}\right)$} \\
\hline $25-30$ & 1.0 & - \\
\hline$<25$ & 1.2 & $0.9-1.5$ \\
\hline $30-35$ & 1.2 & $0.9-1.5$ \\
\hline$>35$ & 1.5 & $1.1-2.1$ \\
\hline \multicolumn{3}{|l|}{ NYHA class } \\
\hline I & 1.0 & - \\
\hline II & 1.3 & $1.0-1.6$ \\
\hline III & 1.2 & $0.9-1.5$ \\
\hline IV & 1.3 & $1.0-1.8$ \\
\hline \multicolumn{3}{|l|}{ Hemoglobin } \\
\hline$<115$ (elective) & 1.2 & $0.7-2.2$ \\
\hline $115-135$ (elective) & 1.1 & $0.9-1.6$ \\
\hline$>135$ (elective) & 1.0 & - \\
\hline$<115$ (in-house) & 1.8 & $1.3-2.6$ \\
\hline 115-135 (in-house) & 1.4 & $0.9-1.9$ \\
\hline$>135$ (in-house) & 1.0 & - \\
\hline$<115$ (urgent) & 2.9 & $1.5-2.5$ \\
\hline 115-135 (urgent) & 1.4 & $0.7-2.7$ \\
\hline$>135$ (urgent) & 1.0 & - \\
\hline$<115$ (emergent) & 1.6 & $0.6-4.0$ \\
\hline 115-135 (emergent) & 1.3 & $0.5-3.2$ \\
\hline$>135$ (emergent) & 1.0 & - \\
\hline \multicolumn{3}{|l|}{ Creatinine (mg/dL) } \\
\hline$<115$ & 1.0 & - \\
\hline $115-140$ & 1.3 & $1.1-1.7$ \\
\hline $140-160$ & 1.6 & $1.1-2.2$ \\
\hline$>160$ & 1.6 & $1.2-2.2$ \\
\hline Preoperative AF & 1.4 & $1.1-1.8$ \\
\hline Diabetes & 1.5 & $1.1-1.8$ \\
\hline $\mathrm{EF}<40 \%$ & 1.3 & $1.0-1.7$ \\
\hline COPD & 1.2 & $0.9-1.5$ \\
\hline CVD & 1.2 & $1.0-1.6$ \\
\hline Hypertension & 1.1 & $0.9-1.5$ \\
\hline Preoperative RF & 1.3 & $0.9-1.9$ \\
\hline \multicolumn{3}{|l|}{ Procedure } \\
\hline CABG & 1.0 & - \\
\hline Valve & 1.2 & $0.9-1.5$ \\
\hline CABG plus valve & 2.3 & $1.8-3.0$ \\
\hline \multicolumn{3}{|l|}{ Status } \\
\hline In-house & 1.1 & $0.9-1.5$ \\
\hline Urgent & 1.8 & $1.8-3.6$ \\
\hline Emergent & 4.3 & $2.9-7.4$ \\
\hline Redo surgery & 1.4 & $0.9-1.9$ \\
\hline \multicolumn{3}{|l|}{ Surgeon } \\
\hline A & 1.0 & - \\
\hline $\mathrm{B}$ & 1.1 & $0.7-1.6$ \\
\hline $\mathrm{C}$ & 1.1 & $0.7-1.6$ \\
\hline $\mathrm{D}$ & 1.1 & $0.6-2.2$ \\
\hline $\mathrm{E}$ & 1.2 & $0.8-1.7$ \\
\hline
\end{tabular}

TABLE 2. Continued

\begin{tabular}{lcc}
\hline Variable & Odds ratio & $\mathbf{9 5} \% \mathbf{~ C I}$ \\
\hline F & 0.7 & $0.5-1.1$ \\
G & 1.1 & $0.6-1.9$ \\
H & 1.1 & $0.8-1.5$ \\
I & 0.8 & $0.5-1.1$ \\
J & 1.1 & $0.8-1.6$ \\
\hline
\end{tabular}

The nested interaction of hemoglobin and status shows the effect of hemoglobin at each level of status. $C I$, Confidence interval; $R F$, renal failure; $M A C E$, Major adverse cardiac events; $B M I$, body mass index; $A F$, atrial fibrillation; $C O P D$, chronic obstructive pulmonary disease; $C V D$, cardiovascular disease; $P V D$, peripheral vascular disease; $E F$, ejection fraction; $N Y H A$, New York Heart Association; $C A B G$, coronary artery bypass grafting.

Simply identifying this novel risk factor as being associated with postoperative events, however, will not, on its own, improve the outcomes for our patients. Because the precursor events are not necessarily preventable, more active efforts are required, after the precursor event, to prevent the postoperative adverse events. Efforts should include quality improvement initiatives designed to increase or improve the system defenses so that they are an impenetrable wall, preventing the precursor event from propagating toward the adverse event.

Our study had a few limitations. First, the low- to medium-risk cohort was selected by truncating the population at the 75th percentile of the predicted risk for postoperative MACE. It might be that as the risk of the patient increases, the effect of a precursor event is less impactful because of the various other risk factors and, consequently, the effect of the precursor events on the occurrence of MACE is blunted. Second, the only measure of time delay of dictation (which would produce a less detailed and more biased operative note) was the surgeon report in the dictation. No other attempt, for practical reasons, was made to validate the time to dictation. As such, late dictations, which would be less likely to report a precursor event, might have been included in the analysis. Assuming that this was a random occurrence, both groups should have been biased equally. Also, the selection of the 4 precursor events was from quality assessment work at our institution and represented events believed common and potentially important at our institution. It could be that we did not include other potentially important events. Some precursor events might behave differently for different procedures. For example, the incidence of regrafting or repair and incomplete revascularization or repair was not different between the 2 groups compared with the mixed population. It might be that this event is more relevant when compared within a single procedure group.

Our study results have demonstrated that precursor events are common and that they affect postoperative adverse events. As the importance of even nominal precursor events becomes clear, efforts aimed at either preventing these events or mitigating their consequences will be essential to decreasing postoperative adverse events. Because 
quality improvement is a cornerstone of cardiac surgery, these types of initiatives are vital in closing the quality improvement loop and providing continued improved outcomes for our patients.

\section{References}

1. Donabedian A. The quality of care: how can it be assessed? JAMA. 1988;260: 1743-8.

2. Donabedian A. Evaluating the quality of medical care: 1966. Milbank Q. 2005; 83:691-729.

3. Janiec M, Sartipy U. Death in low-risk cardiac surgery: Stockholm experience. Interact Cardiovasc Thorac Surg. 2010;11:547-9.

4. Fink AS, Itani KM, Campbell DC Jr. Assessing the quality of surgical care. Surg Clin North Am. 2007;87:837-52. vi.

5. Bier VM, Kunreuther H, Phimister JR. Accident Precursor Analysis and Management: Reducing Technological Risk through Diligence. Washington, DC: National Academies Press; 2004.

6. Wong DR, Vander Salm TJ, Ali IS, Agnihotri AK, Bohmer RM, Torchiana DF. Prospective assessment of intraoperative precursor events during cardiac surgery. Eur J Cardiothorac Surg. 2006;29:447-55.

7. de Leval MR, Francois K, Bull C, Brawn W, Spiegelhalter D. Analysis of a cluster of surgical failures: application to a series of neonatal arterial switch operations. J Thorac Cardiovasc Surg. 1994;107:914-23; discussion 923-4.

8. de Leval MR, Carthey J, Wright DJ, Farewell VT, Reason JT. Human factors and cardiac surgery: a multicenter study. J Thorac Cardiovasc Surg. 2000;119: 661-72.

9. Gaba DM. Human error in anesthetic mishaps. Int Anesthesiol Clin. 1989;27: 137-47.

10. Reason J. Human error: models and management. BMJ. 2000;320:768-70.

11. Reason J. Human Error. New York: Cambridge University Press; 1990.

12. Thomas EJ, Studdert DM, Burstin HR, Orav EJ, Zeena T, Williams EJ, et al. Incidence and types of adverse events and negligent care in Utah and Colorado. Med Care. 2000;38:261-71.

13. Reason J. Understanding adverse events: human factors. Qual Health Care. 1995;4:80-9.

14. Ambler G, Omar RZ, Royston P, Kinsman R, Keogh BE, Taylor KM. Generic, simple risk stratification model for heart valve surgery. Circulation. 2005;112: 224-31.

15. Nashef SA, Roques F, Hammill BG, Peterson ED, Michel P, Grover FL, et al. Validation of european system for cardiac operative risk evaluation (EuroSCORE) in north american cardiac surgery. Eur J Cardiothorac Surg. 2002;22:101-5.

16. Cohen RA. An introduction to PROC LOESS for local regression. In: Proceedings of the Twenty-Fourth Annual SAS Users Group International Conference: April 11-14, 1999, Miami, FL. Cary, NC: SAS Institute Inc; 1999.

17. Hanley JA, McNeil BJ. The meaning and use of the area under a receiver operating characteristic (ROC) curve. Radiology. 1982;143:29-36.

18. Hosmer DW, Lemeshow S. A goodness-of-fit test for the multiple regression model. Commun Statistics. 1980;9:1043-69.

19. Marcin JP, Romano PS. Size matters to a model's fit. Crit Care Med. 2007;35: 2212-3.

20. Harrell FE, Lee KL, Mark DB. Tutorial inBiostatistics multivariable prognostic models: issues in developing models, evaluating assumptions and adequacy, and measuring and reducing errors. Stats Med. 1996;15:361-87.

\section{APPENDIX \\ Model Development}

Variable selection. The candidate variables for model development included the following preoperative characteristics: age, gender, diabetes, frailty, chronic obstructive pulmonary disease, redo sternotomy, atrial fibrillation, hemoglobin, peripheral vascular disease, cerebrovascular disease, creatinine, ejection fraction $<40 \%$, New York Heart Association classification (I-IV), and body mass index. Surgery-related data, such as the urgency of surgery and procedure type, were also included. These candidate variables were chosen a priori and selected through a rigorous review of the published data. ${ }^{1-9,13-15}$

The multicollinearity of the candidate variables was assessed using the variance inflation factor. The linear relationships of the natural variables and their transformations were assessed through locally weighted scatter plot smoothing (LOESS) regression. ${ }^{16}$ The World Health Organization classification of body mass index was used.

\section{Model Evaluation}

Nonparsimonious multivariate logistic regression analysis was used to describe MACE.

Age, gender, procedure type, and surgical priority were chosen a priori and forced into the model. Stepwise selection was implemented for the remainder of the candidate variables. The concordance statistic and $-2 \log$ likelihood were evaluated to assess the contribution of each variable to the model. If a variable did not contribute to an increase in the $\mathrm{C}$ or $-2 \log$ likelihood statistic, it was not retained in the model.

Model discrimination was determined using the concordance statistic. ${ }^{17}$ Model calibration was assessed using the Hosmer-Lemeshow goodness-of-fit statistic ${ }^{18,19}$ and calibration plots. ${ }^{20}$ Deciles of observed and predicted probabilities of MACE were plotted for the calibration plots. ${ }^{5}$ The bootstrap procedure was used to internally validate the model.

\section{Model Performance}

A nonparsimonious multivariate logistic regression analysis was used to describe MACE.

Assessment of the variance inflation for each variable revealed that no variable exceeded 1.5 , allowing all the variables to remain in the final model.

By LOESS regression, the squared transformation of the continuous variable age had the most linear relationship with the log odds of the outcome. Hemoglobin and creatinine had nonlinear relationships with the outcome, despite transformation. The inflection points of the natural variable were taken to create the categorical variables. The hemoglobin inflection points were 115 and 135 and for creatinine were 115,140 , and 160 .

A total of 18 variables remained in the logistic regression model, including a nested interaction of hemoglobin and status. The significant predictors of MACE included variables such as frailty, body mass index $>35 \mathrm{~kg} / \mathrm{m}^{2}$, all creatinine levels, diabetes mellitus, emergent and urgent status, and $\mathrm{CABG}$ plus valve procedure type.

The concordance statistic for the logistic regression was 0.77 , equivalent to a receiver operating characteristic of $77 \%$ (95\% confidence interval, 76-80). The HosmerLemeshow goodness of fit statistic was 0.1218 . 